\title{
Meningococcal Conjugate Vaccine MCV4
}

National Cancer Institute

\section{Source}

National Cancer Institute. Meningococcal Conjugate Vaccine MCV4. NCI Thesaurus. Code C96391.

A conjug ate vaccine of meningococcal polysaccharide used for active immunization against invasive meningococcal disease caused by Neisseria meningiditis serogroups A, C, Y, and W-135. 